\title{
Pre-hospital Care and the Emergency Department
}

\author{
Alex Ritchie, Andrew Imrie, Julia Williams, Alice Cook, \\ and Helen Wilson
}

\subsection{Pre-hospital Care}

Hip fractures are considered a major global healthcare challenge [1] especially in older people with frailty, and this is likely to increase in the next few decades with estimates of the size of the problem being predicted conservatively at 4.5 million cases worldwide by 2050 [2]. The Healthcare Quality Improvement Partnership [3] estimate that in the United Kingdom around 76,000 hip fractures occur every year, and it is reasonable to assume that most of these patients will present through ambulance services; this is a significant number of people requiring assessment, management and treatment from ambulance staff. Whilst there may be differences in scope of clinical practices between (or within) countries relating to pre-hospital care of patients with hip fracture depending on differences in healthcare systems, skill mix of available clinical staff, and use of drugs and technology, the essential principles of management and treatment are likely to be similar.

In the United Kingdom, the first ambulance crew on scene may not include a paramedic. A non-paramedic crew will request paramedic back-up if required for a structured assessment and management of the patient including intravenous drug administration where required.

This chapter is a component of Part 2: Pillar I.

For an explanation of the grouping of chapters in this book, please see Chapter 1: 'The Multidisciplinary Approach to Fragility Fractures Around the World-An Overview'.

\footnotetext{
A. Ritchie $\cdot$ A. Imrie $\cdot$ J. Williams $(\square)$

University of Hertfordshire, Hertfordshire, UK

e-mail: alex.ritchie@nhs.net; a.imrie@herts.ac.uk; j.williams@herts.ac.uk
A. Cook $\cdot$ H. Wilson
Royal Surrey County Hospital NHS Foundation Trust, Guildford, UK
e-mail: Alicecook1@nhs.net; hwilson6@nhs.net


This chapter will focus on principles of management of a patient with suspected hip fracture once any immediate time-critical life-threatening events have been dealt with. This is not to dismiss the severity of a hip fracture in an older person with frailty, as mortality rates within the first year are high (often due to multiple comorbidities) [4]. Pre-hospital management of a patient with an isolated hip fracture, from the point of assimilation of the information gained during the secondary survey, is summarised in Fig. 6.1. This systematic assessment of the patient occurs after completion of a dynamic risk assessment of the scene and a primary survey.

\subsubsection{Clinical Assessment: Primary Survey}

To provide context and clarity, a primary survey in a pre-hospital trauma patient uses a systematic approach such as the $\mathrm{R}<\mathrm{C}>\mathrm{ABCDE}[3,5]$ framework. This involves assessing the patient in a stepwise approach, correcting any complications that present themselves, before moving on to the next stage in the sequence:

- Response level

- Catastrophic Haemorrhage

- Airway (consider c-spine injury at this stage)

- Breathing

- Circulation

- Disability

- Exposure and environment

If the patient is presenting with areas of concern during the primary survey, the ambulance staff will attempt to correct those deficit areas. If this is not possible on scene, they may decide to undertake a time-critical transfer with a pre-alert to an appropriate receiving facility whilst continuing patient treatment en route if it is safe to do so and in accordance with local policies. This may mean that definitive treatment of a patient's hip fracture is delayed, whilst other interventions take a higher priority in, for example, a patient with an acute myocardial infarction or an acute stroke. A systematic primary survey usually takes an experienced paramedic around one minute to complete if no interventions are required during this assessment [5].

\subsubsection{Clinical Assessment: Secondary Survey}

Once it has been established from the primary survey that there is no immediate threat to life, the paramedic (or other attending ambulance staff) will complete a comprehensive secondary survey whilst still being mindful of the need for this patient to get to definitive care in a timely manner. This process includes acquiring a patient history as well as undertaking a 'head to toe' physical assessment and documenting a full set of vital signs. The latter will contribute to the assessment of 


\section{Pre-hospital Management of Suspected Fractured Neck of Femur}

Based on in-depth history and full secondary assessment, the crew identifies

some or all of the following:

- Moderate or severe pain

- Shortened and/or externally rotated leg and/or deformity

- Pain to hip \&/or referred pain to knee

- Unable to straight leg lift

- Unable to weight bear

- Clinician suspects hip fracture

Cautions:

- Has this patient fallen?

- Has this patient had a long-lie prior to assessment?

- Is this patient on anti coagulant or anti platelet theraphy?

- Has this patient had a previous fracture or prosthesis to affected hip?

- Does this patient have a diagnosis of osteoporosis and/or arthritis and/ or malignancy?

\section{Management: Non Paramedic Crew}

- Management of haemorrhage if present

- Check presence and quality of pedal and popliteal pulses

- Monitor vital signs

- Regular assessment of pain levels

- Entonox (or appropriate analgesia within local scope of practice)

- Request Paramedic assistance

- Assess for compromised skin integrity and manage accordingly

- Splinting and/or immobilisation of affected limb

- Optimise patient for surgery, consider as Nil By Mouth

- Extricate patient supine, on a orthopaedic scoop or vacuum mattress

Management: Paramedic Crew

Continue with management identified above, additional elements to consider

- IV Access

- IV Paracetamol (consider IV Morphine, if pain connot be controlled via other methods)

- IV Fluid ( $250 \mathrm{ml}$ bolus Initially)

- Consider Oxygen therapy, to target $94-98 \%$ or $88-92 \%$ in COPD patients post Morphine

- ECG Monitoring - post Morphine

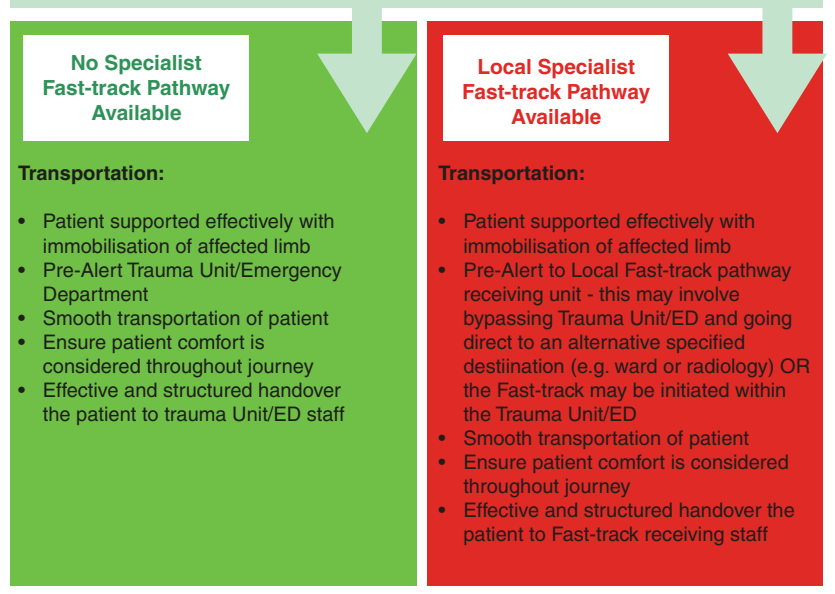

Analgesia Guidelines:

Refer to local policies

Mild - Moderate Pain

- Endonox, PRN - IH

- Methoxyflurane, $3 \mathrm{ml}$ - IH

- Paracetamol, $1 \mathrm{~g}$ - IV

Severe Pain

- Morphine, 2-10mg - IV

- Ondansetron, 4mg - IV (or alternative anti-emetic for prophylactic prevention of nausea)

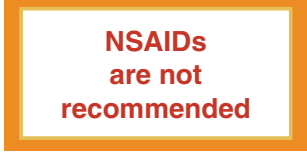

REMEMBER

Reassess patient's pain at all stages of treatment. Consider non-

pharmacological pain

management techniques

such as positioning and

physical support of limbs

Would this patient be suitable for Fascia Iliaca

Compartment Block (1)

(FICB)?

Contraindications of FICB:

- Patient declines

- Allergy to local anaesthetic

- Infection at injection site

- Previous femoral vascular surgery

- Patient connot report complications (eg. severe dementia, confusion)

- High risk of compartment syndrome

If FICB contraindicated,

consider IV Morphine - as per local policy

(1) At the time of writing, paramedic administered FICB is not standard practice in the UK

Fig. 6.1 Pre-hospital management of a suspected fractured neck of femur post-secondary survey 
the patient's National Early Warning Score 2 (NEWS2) which will provide baseline information for the hospital to monitor any changes in the patient's condition.

\subsubsection{Patient History}

As part of the secondary survey, gaining a patient history as soon as possible after the fall is likely to give the most accurate account. History taking should be structured and include details of the fall and events leading up to it, the past medical history, allergies and medications and a social history [5, 6]. Global characteristics of the patient and their environment are noted, including general appearance, living arrangements and level of independence. This information is essential to support subsequent discharge planning.

Patients with a fall and a 'long lie' [7], which is considered anything in excess of one hour, are at risk of hypothermia, compromised skin integrity due to unrelieved pressure, rhabdomyolysis and aspiration pneumonia [7-9].

Early pain assessment and management is a key role for ambulance staff [10]. Classically, in patients with a hip fracture, there is pain on movement of the leg, in the groin and/or thigh, with pain referred to the knee [11].

Establishing a list of current medications and any known drug allergies is important for ongoing management. Recognition of anti-platelet or anticoagulant therapy is essential. Ambulance staff need to establish whether the patient has taken any analgesics recently, before administration of further doses.

\subsubsection{Physical Assessment and Vital Signs}

Early establishment of the patient's vital signs is recommended to identify possible intercurrent illness, monitor the patient's progress and recognise any deterioration in their clinical status. Basic observations should include blood glucose testing to rule out hypoglycaemia. The assessment of the limb should include inspection and palpation, comparing it to the uninjured side [6], examining for irregularities/deformities, swelling or bruising. Classically, with a hip fracture there is shortening and external rotation of the leg. Undisplaced hip fractures may have no signs, but patients may complain of pain on internal rotation and will be unable to straight-leg raise the affected limb [12]. Patients may be able to get up but then are unable to weight bear due to pain. In older people presenting with these signs and symptoms, ambulance crews should have a low threshold for transferring the patient to definitive care for radiological assessment.

If the emergency call relates to a fall where no clear extrinsic reason can be identified, then a 12-lead ECG should be performed, and a brief neurological assessment such as FAST (Face/Arm/Speech Test) should be undertaken to exclude stroke/TIA [7].

This information will provide the paramedic with relevant information to develop differential diagnoses and formulate a management plan whilst always being mindful that time is a factor for these patients and extended time on scene if they have a hip fracture may not be beneficial to their overall outcomes. 


\subsubsection{Management of Pain}

Pain management should include non-pharmacological options such as splinting, immobilisation and positioning in addition to the use of pharmacological agents, always with continual assessment of the level of the patient's pain.

Consideration needs to be given to both static pain (when the patient is at rest) and dynamic pain (when the patient is moving or being moved). Adequate analgesia should be given prior to moving the patient when transferring them from the scene of the injury to the ambulance and during transportation to hospital. Pain rating scales may be used to assess the efficacy of any drugs or techniques used to control and reduce pain. There are many different pain scales available [5-7, 13], but broadly there are Numeric Rating Scales (NRS) where the patient is asked to score their pain between 0 and 10 with 10 being the 'worst level of pain'; Visual Analogue Scales (VAS) where patients choose any point on a line usually between 0 and 100 with 0 being 'no pain' and 100 being the 'worst level of pain'; Faces Pain Scale (frequently used with children and people with some level of cognitive impairment) where the faces at one end of the continuum are happy faces and then gradually change to faces in torment; and a Verbal Rating Scale (VRS) where people align with statements such as 'No Pain', 'Mild Pain' etc. [14].

Whatever scale is used, it is essential to assess pain levels both before and after intervention to ensure effective management.

Choice of analgesia may in the first instance depend on the skill mix of the ambulance crew. In the United Kingdom, if a non-paramedic crew is in attendance the most usual quick acting analgesic offered to the patient is likely to be Entonox which was introduced into UK ambulance services in 1970 [15]. Entonox is fast acting and rapidly excreted, but there can be some challenges to its use with older patients with frailty. The delivery device for Entonox is a mouthpiece and a demand valve which requires respiratory effort and coordination; this is not suitable for all patients. The alternative delivery route is via a face mask [16] which can be considered if patients struggle with the mouthpiece. Ideally, the patient should be able to hold the mask independently with Entonox being self-administered, but again this is not possible for all patients. If a paramedic is available, then intravenous paracetamol should be considered, as it has been shown to be comparable in analgesic effect to that of morphine when administered for pain control in isolated limb trauma [17]. It is important to ensure that intravenous paracetamol is given at appropriate doses in low-weight patients.

Another approach to managing pain in isolated hip fracture is paramedicadministered Fascia Iliaca Compartment Block (FICB) delivered in the pre-hospital setting. This is a new consideration in relation to paramedics' scope of practice and it is not yet routine in the United Kingdom. FICB is covered in more depth later in this chapter. A feasibility trial of paramedic-administered FICB has been carried out in Wales [18], and the early findings are positive; funding has been approved for a fully powered multi-centre randomised controlled trial of paramedic-administered FICB in pre-hospital settings starting in October 2020.

If the patient's pain is not controlled, and if there is a paramedic available, then intravenous morphine may be considered. This should be started at the lowest 
possible dose and titrated to therapeutic effect. It should be emphasised that opiates are often associated with significant side effects particularly in older patients with frailty and are not recommended as the drug of first choice in this age group [7]. Concurrent prophylactic use of an anti-emetic is recommended due to high incidence of nausea associated with opioids [5]. Careful observation of vital signs should be undertaken post administration of intravenous morphine, as it is a central nervous system suppressant. ECG and blood pressure monitoring are advised to detect any early changes. Additionally, the patient may need supplemental oxygen post-morphine administration, as it is a respiratory depressant. Target oxygen saturation in adults is between $94 \%$ and $98 \%$ [7] unless they are diagnosed with COPD when target saturations will usually range from $88 \%$ to $92 \% \mathrm{SPO}_{2}$.

Non-pharmacological techniques of pain management include positioning the patient carefully and ensuring limbs are well supported. Splinting and immobilisation can help to reduce pain as well as protect from further internal damage to blood vessels and/or nerves. Current recommendations are to use padding between the legs for the whole length of the leg and then to use broad fold bandages. The bandages should be applied in sequence with a figure of eight around the feet and ankles being placed first of all. This will help with gentle manual traction to straighten the leg and bring it into position. Then, there should be two broad fold bandages placed above the knee and two below the knee to securely keep the legs in alignment and minimise movement [7].

Successful pain management for these patients is likely to employ a combination of pharmacological and non-pharmacological approaches.

\subsubsection{Fluid Replacement}

Older patients with frailty are often dehydrated and should be given intravenous fluids. Caution needs to be exercised in patients with a history of heart failure. In the United Kingdom, the recommendations recommend a loading volume of $250 \mathrm{mls}$ of crystalloids in the form of intravenous sodium chloride $0.9 \%$ [7] to improve tissue perfusion and combat dehydration, but each patient's needs will be different. Another indication for administration of intravenous fluids is related to trauma and possible haemorrhage. Paramedics need to be guided by local policies and practices relating to fluid replacement therapy, as these will determine what options they have in management of the haemodynamic status of the patient [5-7, 19].

Traditionally, patients have been kept nil by mouth in anticipation of surgery, but most units would now advocate feeding the patient until a definite time for surgery is confirmed, to avoid long periods of starvation.

\subsubsection{Extrication}

Ambulance staff must consider the best method of transferring the patient with a hip fracture into the ambulance and transporting them to a place of definitive care. A 
carry chair is not appropriate with the risk of further damage at the site of injury and is likely to be extremely painful for the patient [19]. An orthopaedic scoop stretcher is a good option to get a patient from the floor onto a trolley without the need to log-roll the patient. If the patient has to be taken downstairs, however, the scoop stretcher may be more difficult to use, and the crew may need to request additional staff to do this safely. Deployment of a vacuum mattress can also be considered as an adjunct to extrication, but, at times, there may be limited options [19].

\subsubsection{Transportation}

Fast-track hip fracture care has been developed in some countries, but there is no standardisation. Further research is required to identify which components, at what stage, and in what combination of the fast-track pathway have the most significant impact on patients' outcomes and experiences [20]. This should include research in pre-hospital care, involving paramedics and other ambulance staff.

In the United Kingdom, there are a growing number of fast-track pathways for patients who have a suspected hip fracture. In some regions, after a pre-alert call from the ambulance crew to the hospital, patients will by-pass the Emergency Department and go straight to radiology or a specialist ward to reduce time to definitive treatment. Other areas rely on the pre-alert call from the ambulance staff to the receiving unit to ensure the patient is fast-tracked through the Emergency Department [7].

Key principles of patient management during transportation revolve around continual monitoring of the patient's vital signs. Equally important is to re-assess pain levels and try to minimise exposure to protracted pain through a combination of appropriate pharmacological interventions in conjunction with effective splinting and immobilisation of the limb, comfortable positioning of the patient and, as far as is possible, a smooth, controlled approach to driving to avoid the patient being exposed to unnecessary sudden movements and jolts.

Effective handover to the receiving unit staff is essential and is frequently performed using various frameworks such as IMIST-AMBO [21] (Identification of patient, Mechanism of injury/medical complaint, Injuries/information relative to the complaint, Signs, vitals and GCS, Treatment/response to treatment, Allergies, Medications, Background history and Other (social) information), developed in Australia. It has been shown, amongst other things, to improve the quality of information, shorten the duration of handover and reduce the number of questions asked after the handover. In the United Kingdom, ATMIST (Age, Time of onset, Medical complaint/injury, Investigation, Signs and Treatment) is the recommended mnemonic [7] which contains the relevant clinical information for an effective handover. There is still a need for further evidence to establish whether standardised frameworks consistently achieve improved performance in this area and, if so, to what degree [22].

Pre-hospital clinicians have an important role in the management of patients with hip fracture. They are well placed to get a clear account of the patient's fall, comorbidities and circumstances and handover of this information to the hospital team can 
save time and repetition. Early appropriate management of pain and hydration can also influence peri-operative complications. It is important that pre-hospital staff and hospital staff work and learn together, to continually improve patient care.

\subsection{The Emergency Department}

As soon as a patient with suspected hip fracture arrives, triage assessment should ensure that the patient is cardiovascularly stable, using an early warning score. A brief review of the cause of the fall is essential to ensure that unstable medical conditions such as dehydration, sepsis, gastro-intestinal haemorrhage, stroke or cardiac syncope are not missed.

Most hospitals in the United Kingdom now have a fast-track pathway for patients with suspected hip fracture [23]. This enables patients who are stable at triage to be prioritised for X-ray. The current targets from the Royal College of Emergency Medicine are that $90 \%$ of X-rays completed should be within 60 minutes of arrival and $75 \%$ of patients with a hip fracture should be referred within 120 minutes of arrival [24].

For patients with frailty or those with a history of respiratory disease, a baseline chest X-ray should be performed at the same time. In patients with known malignancy or concerns about pathological fracture, a full-length femur view is required to assess for lytic lesions and plan surgery.

Blood should be taken early for routine testing, ideally with point-of-care testing of haemoglobin and lactate to dictate early management. A significant proportion of patients will require blood transfusion in the peri-operative period, and group-andsave samples should be sent routinely.

Those with complex fractures or those on anti-platelets or anticoagulants may bleed significantly into the fracture site, and resuscitation with intravenous fluids should be commenced early, with consideration of blood transfusion in appropriate cases.

\subsubsection{Nutrition and Hydration}

Patients should be encouraged to eat and drink if able, until 6 hours before planned surgery. For elective patients, there is evidence to suggest that it is safe to continue with clear oral fluids up until 2-3 hours pre-surgery [25]. Oral carbohydrate loading drinks are actively encouraged for elective patients with evidence from enhanced recovery programmes. However, care must be taken with frail older patients with fragility fractures, as some will have required opiates for pain control and may have delayed gastric emptying.

Most patients with frailty are at high risk of malnutrition and should not be kept nil by mouth unnecessarily. Those who have had a long lie after their fall, or who have intercurrent illness, are likely to be dehydrated, and this should be addressed with appropriate fluid resuscitation. Others with pain or delirium may not manage adequate oral fluids and should have maintenance intravenous fluids prescribed. 
Caution must be taken in those with decompensated heart failure or fluid overload from other conditions.

\subsubsection{Management of Pain}

Pain from a fracture is best managed by initial immobilisation and fixation where possible to aid healing. Immobilisation in plaster or with traction without surgery has significant risks in frail patients, as skin integrity is often poor. Immobility often rapidly leads to poor oral intake, generalised muscle weakness, orthostatic pneumonia, thromboembolic disease, incontinence and skin breakdown. As such, a decision for early surgery is usually the best option.

\subsubsection{Ongoing Analgesia}

Pain leads to distress and is the symptom that people fear the most. It may be a key feature in the development of delirium in those at risk.

As previously discussed, it is important to monitor pain both at rest (static pain) and on movement (dynamic pain), as even those immobilised still experience pain during personal care and toileting. Pain should be measured, ideally using a validated score on admission and after 30 minutes of administering analgesia, to ensure effectiveness [26]. Ongoing review of pain should form part of routine nursing observations. Paracetamol should be continued every 6 hours. It is reported to have very few side effects and may also be effective in reducing delirium [27].

Opioids such as Codeine, Tramadol and others have significant side effects and are poorly tolerated by older people causing nausea, vomiting, constipation and confusion and should be avoided.

Opiates may be required but should be used in the lowest possible dose to avoid nausea, vomiting, sedation and respiratory depression. Older patients with poor renal function may not metabolise opiates effectively, and even small doses can cause prolonged side effects.

Non-steroidal anti-inflammatory drugs (NSAID) should only be used with extreme caution. Trauma and poor oral intake increase the risk of gastric irritation and bleeding and this may be exacerbated with the use of NSAIDs. Those on antihypertensive medication are at high risk of renal impairment with NSAIDs.

\subsubsection{Local Nerve Blocks}

Local nerve blocks are increasingly being used to manage both static and dynamic pain and to reduce the requirements for opiate analgesia.

NICE guidelines state that nerve blocks should be used where possible to limit the use of systemic analgesia [28]. For hip fracture, both femoral nerve blocks and fascia-iliaca compartment blocks (FICB) have been shown to be effective [29]. 
Single dose fascia-iliac a blocks given in the pre-operative period significantly reduce the post-operative and total analgesic requirements. These patients experience lower rates of delirium and those that manage to return directly home, do so more quickly, reducing the cost of inpatient stay but also lessening the burden of hip fracture on older comorbid patients [30].

Traditionally performed as part of the anaesthetic, these procedures are being used earlier to manage pre-operative pain in the first 8-16 hours. FICB is a lowskill, inexpensive procedure which may be performed by trained individuals including non-physician practitioners as outlined in a position statement by the Association of Anaesthetists of Great Britain and Ireland [31]. The fascia-iliaca compartment is a potential space containing the iliacus muscle, the femoral nerve and the lateral cutaneous nerve of the thigh. A single high-volume injection using $30 \mathrm{mls}$ (for under $55 \mathrm{~kg}$ ) to $40 \mathrm{mls}$ (over $55 \mathrm{~kg}$ ) of $0.25 \%$ levobupivacaine local anaesthetic, injected through the fascia lata and into the fascia iliaca compartment, will affect the femoral nerve, the lateral femoral cutaneous nerve and, to some extent, the obturator nerve. These supply the medial, anterior and lateral aspects of the thigh and the femoral head. Although this can be performed without ultrasound by trained practitioners with good effect, a small study suggests that ultrasound guidance, where available, may improve efficacy from $47 \%$ to $60 \%$ up to $82-95 \%$ [32].

Guidelines recommend that observations need to be performed for 30 minutes following a nerve block. As the fracture pain is relieved, the side effects of previously administered morphine may become apparent as respiratory depression, with fatal consequences [33].

\subsubsection{Skin Care}

Traction is rarely used in hip fracture, as it offers little benefit and is poorly tolerated in those with frail skin.

There is a high risk of skin breakdown and pressure ulcers in those who are immobile, particularly in those with low body weight, malnutrition, poor skin and incontinence. Patients with diabetes or neuropathy have an increased risk.

Pressure-relieving mattresses should be used where available to minimise risk of pressure damage.

Urinary catheters may be considered for short-term use pre-operatively to help to minimise skin damage and reduce the need for painful movement.

\subsubsection{Referral for Early Surgery}

An orthopaedic assessment should occur as soon as the X-rays are available, and if a fracture is confirmed, a proposed time for an early operation should be agreed. 


\subsection{Summary}

In general, patients with hip fracture need early assessment and initial management of pain. This can be initiated in the pre-hospital setting to reduce distress associated with transfer to hospital. The principles of assessment, management and optimisation have an increasing evidence base, but the patient pathway may vary with different roles of healthcare practitioners in different healthcare systems.

\section{References}

1. Lu Y, Uppal HS (2019) Hip fractures: relevant anatomy, classification, and biomechanics of fracture and fixation. Geriatr Orthop Surg Rehabilit 10:2151459319859139. https://doi. org/10.1177/2151459319859139

2. Veronese N, Maggi S (2018) Epidemiology and social costs of hip fracture. Injury 49(8): $1458-1460$

3. Healthcare Quality Improvement Partnership. National Hip Fracture Database Annual Report. https://data.gov.uk/dataset/3a1f3c15-3789-4299-b24b-cd0a5b1f065b/national-hip-fracturedatabase-annual-report-2018. Accessed 23 Dec 2019

4. Riemann AHK, Hutchison JD (2016) The multidisciplinary management of hip fractures in older patients. Orthop Traumatol 30(2):117-122

5. Willis S, Dalrymple R (eds) (2020) Fundamentals of paramedic practice: a systems approach, 2nd edn. Wiley Blackwell, Oxford

6. Gregory P, Ward A (2010) Sanders' paramedic textbook. Mosby, Edinburgh

7. Joint Royal Colleges Ambulance Liaison Committee (2019) Association of Ambulance Chief Executives JRCALC Clinical Guidelines 2019. Class Professional Publishing, Bridgewater

8. Wongrakpanich S, Kallis C, Prasad P, Rangaswami J, Rosenzweig A (2018) The study of rhabdomyolysis in the elderly: an epidemiological study and single center experience. Aging Dis 9(1): $1-7$

9. Bledsoe BE, Porter RS, Cherry RA (2014) Paramedic care, principles \& practice, 4th edn. Pearson Education Ltd, New York

10. Pilbery R, Lethbridge K (2016) Ambulance care practice. Class Professional Publishing, Bridgewater

11. LeBlanc KE, Muncie HL Jr, LeBlanc LL (2014) Hip fracture: diagnosis, treatment, and secondary prevention. Am Fam Physician 89(12):945-951

12. Greaves I, Porter K (2007) Oxford handbook of pre-hospital care. Open University Press, Oxford

13. Blaber AY, Harris G (eds) (2016) Assessment skills for paramedics, 2nd edn. Open University Press, Maidenhead

14. Ferreira-Valente MA, Pais-Ribeiro JL, Jensen MP (2011) Validity of four pain intensity rating scales. Pain 152(10):2399-2404

15. Baskett PJ, Withnell A (1970) Use of Entonox in the ambulance service. Br Med J 2:41-43

16. Gregory P, Mursell I (2010) Manual of clinical paramedic procedures. Wiley, Oxford

17. Craig M, Jeavons R, Probert J et al (2012) Randomised comparison of intravenous paracetamol and intravenous morphine for acute traumatic limb pain in the emergency department. Emerg Med J 29(1):37-39

18. Jones JK, Evans BA, Fegan G, Ford S, Guy K, Jones S et al (2019) Rapid Analgesia for Prehospital hip Disruption (RAPID): findings from a randomised feasibility study. Pilot Feasibil Stud 5(1):77

19. Eaton G (2012) Management of an isolated neck-of-femur fracture in an elderly patient. JPP 4(7):400-408 
20. Pollmann CT, Røtterud JH, Gjertsen JE, Dahl FA, Lenvik O, Årøen A (2019) Fast track hip fracture care and mortality-an observational study of 2230 patients. BMC Musculoskelet Disord 20(1):248

21. Iedema R, Ball C, Daly B, Young J, Green T, Middleton PM, Foster-Curry C, Jones M, Hoy S, Comerford D (2012) Design and trial of a new ambulance-to-emergency department handover protocol: 'IMIST-AMBO'. BMJ Qual Saf 21(8):627-633

22. Fitzpatrick D, McKenna M, Duncan EA, Laird C, Lyon R, Corfield A (2018) Critcomms: a national cross-sectional questionnaire based study to investigate prehospital handover practices between ambulance clinicians and specialist prehospital teams in Scotland. Scand J Traum Resuscit Emerg Med 26(1):45

23. Audit Commission (2000) United they stand: co-ordinating care for elderly patients with hip fractures. HMSO, London

24. Royal College Emergency Medicine (2014) Clinical Standards for Emergency Departments. https://www.rcem.ac.uk/docs/Clinical\%20Standards\%20and\%20Guidance/Clinical\%20 Standards\%20for\%20Emergency\%20Departments.pdf

25. Brady M, Kinn S, Stuart P (2003) Preoperative fasting for adults to prevent peri-operative complications. Cochrane Database Syst Rev 2003(4):CD004423

26. NICE (2011) NICE clinical guideline 124. Hip Fracture: the management of hip fracture in adults. Guidance.nice.org.uk/cg124

27. Morrison R, Magaziner J, McLaughlin MA et al (2003) The impact of post-operative pain on outcomes following hip fracture. Pain 103(3):303-311. Management of Pain Reduces Delirium.

28. NICE Guidance: The Management of Hip Fractures in Adults (2017) Page 36. https://www. nice.org.uk/guidance/cg124/evidence/full-guideline-pdf-183081997

29. Foss NB, Kristensen BB, Bundgaard M et al (2007) Fascia iliaca compartment blockade for acute pain control in hip fracture patients: a randomized, placebo-controlled trial. Anesthesiology 106(4):773-778

30. Callear J, Shah K (2016) Analgesia in hip fractures: Do fascia-iliac blocks make any difference? BMJ Quality Improv Rep 5:u210130.w4147

31. Griffiths R, Tighe S (2013) Fascia iliaca blocks and non-physician practitioners. Aagbi Position Statement. http://www.aagbi.org/sites/default/files/Fascia\%20Ilaica\%20statement\%20 22JAN2013.pdf

32. Dolan J et al (2008) Ultrasound guided fascia iliaca block: a comparison with the loss of resistance technique. Reg Anesth Pain Med 33(6):526-531

33. RCEM (2018) Safety alert: the importance of monitoring after Fascia-iliaca block. https:// www.rcem.ac.uk/docs/Safety $\% 20$ Resources $\% 20+\% 20$ Guidance/RCEM_Fascia\%20 Iliaca\%20Block_Safety\%20Newsflash\%20Feb\%20(22022018)\%20revised.pdf

Open Access This book is licensed under the terms of the Creative Commons AttributionNonCommercial-NoDerivatives 4.0 International License (http://creativecommons.org/licenses/ by-nc-nd/4.0/), which permits any noncommercial use, sharing, distribution and reproduction in any medium or format, as long as you give appropriate credit to the original author(s) and the source, provide a link to the Creative Commons license and indicate if you modified the licensed material. You do not have permission under this license to share adapted material derived from this book or parts of it.

The images or other third party material in this book are included in the book's Creative Commons license, unless indicated otherwise in a credit line to the material. If material is not included in the book's Creative Commons license and your intended use is not permitted by statutory regulation or exceeds the permitted use, you will need to obtain permission directly from the copyright holder.

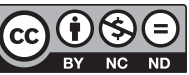

